\title{
Prenatal diagnosis and risk factors for preoperative death in neonates with single right ventricle and systemic outflow obstruction: Screening data from the Pediatric Heart Network Single Ventricle Reconstruction Trial*
}

\author{
Andrew M. Atz, MD, ${ }^{\mathrm{a}}$ Thomas G. Travison, PhD,${ }^{\mathrm{b}}$ Ismee A. Williams, MD, MS, ${ }^{\mathrm{c}}$ Gail D. Pearson, MD, ScD, ${ }^{\mathrm{d}}$ \\ Peter C. Laussen, MBBS, ${ }^{\mathrm{e}}$ William T. Mahle, MD, ${ }^{\mathrm{f}}$ Amanda L. Cook, MD, ${ }^{\mathrm{g}}$ Joel A. Kirsh, MD, ${ }^{\mathrm{h}}$ \\ Mark Sklansky, MD, ${ }^{\mathrm{i}}$ Svetlana Khaikin, RN, MPH, ${ }^{\mathrm{h}}$ Caren Goldberg, MD ${ }^{\mathrm{j}}$ Michele Frommelt, MD, ${ }^{1}$ \\ Catherine Krawczeski, MD, ${ }^{\mathrm{m}}$ Michael D. Puchalski, MD, ${ }^{\mathrm{n}}$ Jeffrey P. Jacobs, MD, ${ }^{\mathrm{o}}$ Jeanne M. Baffa, MD, ${ }^{\mathrm{p}}$ \\ Jack Rychik, MD, ${ }^{\mathrm{q}}$ and Richard G. Ohye, MD, ${ }^{\mathrm{k}}$ for the Pediatric Heart Network Investigators
}

Objectives: The purpose of this analysis was to assess preoperative risk factors before the first-stage Norwood procedure in infants with hypoplastic left heart syndrome and related single-ventricle lesions and to evaluate practice patterns in prenatal diagnosis, as well as the role of prenatal diagnosis in outcome.

Methods: Data from all live births with morphologic single right ventricle and systemic outflow obstruction screened for the Pediatric Heart Network's Single Ventricle Reconstruction Trial were used to investigate prenatal diagnosis and preoperative risk factors. Demographics, gestational age, prenatal diagnosis status, presence of major extracardiac congenital abnormalities, and preoperative mortality rates were recorded.

Results: Of 906 infants, $677(75 \%)$ had prenatal diagnosis, $15 \%$ were preterm $(<37$ weeks' gestation), and $16 \%$
were low birth weight $(<2500 \mathrm{~g})$. Rates of prenatal diagnosis varied by study site $(59 \%$ to $85 \%, P<.0001)$. Ma-
jor extracardiac congenital abnormalities were less prevalent in those born after prenatal diagnosis $(6 \%$ vs $10 \%$,
$P=.03)$. There were $26(3 \%)$ deaths before Norwood palliation; preoperative mortality did not differ by prenatal
diagnosis status $(P=.49)$. In multiple logistic regression models, preterm birth $(P=.02)$, major extracardiac
congenital abnormalities $(P<.0001)$, and obstructed pulmonary venous return $(P=.02)$ were independently
associated with preoperative mortality.

Conclusions: Prenatal diagnosis occurred in $75 \%$. Preoperative death was independently associated with preterm birth, obstructed pulmonary venous return, and major extracardiac congenital abnormalities. Adjusted for gestational age and the presence of obstructed pulmonary venous return, the estimated odds of preoperative mortality were 10 times greater for subjects with a major extracardiac congenital abnormality. (J Thorac Cardiovasc Surg 2010;140:1245-50)

Diagnosis of congenital heart disease with fetal echocardiography was first reported in $1984,{ }^{1}$ and clinical use has been growing steadily. ${ }^{2,3}$ Hypoplastic left heart syndrome (HLHS) and other related morphologic single right ventricular lesions with systemic outflow obstruction are

\footnotetext{
From the Division of Pediatric Cardiology, ${ }^{\mathrm{a}}$ Medical University of South Carolina, Charleston, SC; New England Research Institutes, ${ }^{\mathrm{b}}$ Watertown, Mass; Division of Pediatric Cardiology, ${ }^{\mathrm{c}}$ Columbia University Medical Center, New York, NY; Division of Cardiovascular Diseases, ${ }^{\mathrm{d}}$ National Heart, Lung, and Blood Institute, Bethesda, Md; Department of Cardiology, e Children's Hospital Boston, Boston, Mass; Division of Pediatric Cardiology, ${ }^{\mathrm{f}}$ Emory University, Atlanta, Ga; Division of Pediatric Cardiology, ${ }^{\mathrm{g}}$ Wake Forest University, Winston-Salem, NC; Division of Cardiology, ${ }^{\mathrm{h}}$ Hospital for Sick Children, Toronto, Ontario, Canada; Division of Cardiology, ${ }^{i}$ Children's Hospital Los Angeles, Los Angeles, Calif; Departments of Pediatrics and Communicable Disease ${ }^{\mathrm{j}}$ and Department of Surgery, ${ }^{\mathrm{k}}$ University of Michigan, Ann Arbor, Mich; Division of Cardiology, ${ }^{1}$ Children's Hospital of Wisconsin, Milwaukee, Wis; Division of Cardiology, ${ }^{\mathrm{m}}$ Cincinnati Children's Hospital Medical Center, Cincinnati, Ohio; Division of Pediatric Cardiology, ${ }^{\mathrm{n}}$ Primary Children's Medical Center and The University of Utah, Salt Lake City, Utah; Division of Pediatric Heart Surgery, ${ }^{\circ}$ The Congenital Heart Institute of Florida, St. Petersburg, Fla; Division of Pediatric Cardiology, ${ }^{\mathrm{p}}$ Nemours Cardiac Center, Alfred I. DuPont
}

among the most commonly prenatally diagnosed congenital heart defects. ${ }^{4}$ Neonates with HLHS are often critically ill on de novo presentation and must be resuscitated and stabilized with prostaglandin $\mathrm{E}$ infusion before initial palliative surgery can be considered. Therefore the assumption has

Hospital for Children, Wilmington, Del; and Division of Cardiology ${ }^{\mathrm{q}}$ Children' Hospital of Philadelphia, Philadelphia, Pa.

Supported by U01 grants from the National Heart, Lung, and Blood Institute (HL068269, HL068270, HL068279, HL068281, HL068285, HL068292, HL068290, HL068288, and HL085057).

Disclosures: None.

* Participants in the Pediatric Heart Network's Single Ventricle Reconstruction Trial are shown in Appendix 1.

Received for publication Jan 10, 2010; revisions received April 27, 2010; accepted for publication May 16, 2010; available ahead of print June 21, 2010.

Address for reprints: Andrew M. Atz, MD, Department of Pediatric Cardiology, Medical University of South Carolina, 171 Ashley Ave, Charleston, SC 29425 (E-mail: atzam@musc.edu).

0022-5223/\$36.00

Copyright (C) 2010 by The American Association for Thoracic Surgery doi:10.1016/j.jtcvs.2010.05.022 


\section{Abbreviation and Acronym \\ HLHS = hypoplastic left heart syndrome}

been that prenatal diagnosis should improve outcome, but most studies have not demonstrated an improvement in postoperative survival with prenatal diagnosis. ${ }^{5,6}$ A single study found that prenatal diagnosis was associated with improved preoperative clinical status and improved survival after the first-stage reconstruction (the Norwood procedure). ${ }^{7}$ Considerable effort has been expended to identify risk factors for postoperative mortality after Norwood palliation. ${ }^{8,9}$ However, some infants die before reaching the operating room, and risk factors for preoperative mortality and potential associations among prenatal diagnosis, patients' characteristics, and preoperative mortality are poorly understood. The aims of this investigation were to describe the following:

- current prevalence and timing of diagnosis (prenatal vs postnatal) in a large, contemporary multicenter cohort of patients with HLHS or related morphologic single right ventricular lesions with systemic outflow obstruction;

- center-level variation in the rate of prenatal diagnosis;

- patients' characteristics associated with prenatal diagnosis; and

- determinants of preoperative mortality, including patients' characteristics and prenatal diagnosis.

\section{MATERIALS AND METHODS \\ Design of the Single Ventricle Reconstruction Trial}

Data for the analyses were obtained as part of a screening protocol used in a multicenter randomized trial of modified Blalock-Taussig shunt versus right ventricular-pulmonary artery shunt introduced during a stage I palliation (Norwood) procedure. All live births with a diagnosis of a single morphologic right ventricle with systemic outflow obstruction were eligible for inclusion in the trial. The trial's primary outcome measure was the composite proportion of subjects experiencing death or cardiac transplantation 12 months after randomization. The design of this trial, conducted by the National Heart, Lung, and Blood Institute-funded Pediatric Heart Network has been previously described. ${ }^{10}$ From May 2005 to July 2009, 15 centers enrolled 555 subjects (ClinicalTrials.gov number: NCT00115934).

The protocol was approved by an independent protocol review committee and data and safety monitoring board, by institutional review boards at each clinical center, and at the data coordinating center. all centers followed the same protocol and study procedures.

\section{Screening of Potential Subjects}

During the enrollment period, all neonates with a morphologic single right ventricle and systemic outflow obstruction admitted to participating centers were assessed for inclusion in the trial. Data collected at screening included birth weight, race, sex, gestational age, fetal intervention (atrial septostomy or aortic valve dilation), detailed cardiac diagnosis, presence of major extracardiac congenital abnormalities or acquired extracardiac disorders, and presence of and age at prenatal diagnosis. For the purposes of this study, obstructed pulmonary venous return was defined by the use of postnatal intervention, including balloon septostomy, open atrial septectomy, or urgent Norwood procedure. Preoperative mortality was also recorded.

For the purposes of analysis, extracardiac congenital abnormalities were divided into chromosomal and nonchromosomal abnormalities. Chromosomal abnormalities included trisomies 13, 18, and 21; Turner's syndrome, Ellis-van Creveld syndrome; Goldenhar syndrome; Scimitar syndrome; Jacobsen syndrome; and other unidentified chromosomal abnormalities or genetic syndromes. Nonchromosomal abnormalities included acquired extracardiac disorders (eg, meconium aspiration with need for highfrequency ventilation and persistent renal failure requiring dialysis) that the site investigator considered could independently affect the likelihood of the subject meeting the primary end point of death or transplantation at 1 year. $^{10}$

\section{Analytic Sample and Statistical Analyses}

Statistical analyses were performed with SAS software version 9.2 (SAS Institute, Inc, Cary, NC) and the R System, version 2.8.1 (R Foundation for Statistical Computing, Vienna, Austria). Exploratory analyses incorporating graphic and tabular displays were used to assess bivariate associations. Sample means, medians, and proportions accompanied by $95 \%$ confidence intervals were used to provide descriptive summaries. $\chi^{2}$ Tests of equality of proportions and Fisher's exact and Student's $t$ tests of equality of means were used to formally test hypotheses of no differences in various factors versus prenatal diagnosis status. Simple and multiple logistic regressions were used to assess the relative strength of association between multiple risk factors and preoperative death. Backwards covariate selection with a significance criterion of 0.05 was used to construct the multivariate logistic regression model. Analysis of variance and $\chi^{2}$ statistics were used to assess the degree of cross-center variation in the proportion of subjects with prenatal diagnosis and, among those subjects with prenatal diagnosis, fetal gestational age at that time. An adjusted screening population size was used for 2 centers that had abridged screening periods of participation to obtain center volume estimates based on total trial duration.

\section{RESULTS}

\section{Characteristics Associated With Prenatal Diagnosis}

From May 2005 to July 2009, 15 centers screened 921 neonates for the Single Ventricle Reconstruction Trial; 15 were determined to not meet the study entry criteria of morphologic single right ventricle with systemic outflow obstruction and were excluded, leaving a total of 906 subjects for this analysis. Prenatal diagnosis was made in 677 (75\%) of 906 subjects. Unadjusted associations between subjects' risk factors and prenatal diagnosis are presented in Table 1. The sex and racial/ethnic distributions of subjects with and without prenatal diagnosis were similar. Although the proportion of preterm infants did not differ based on prenatal diagnosis status, subjects without a prenatal diagnosis were on average born at a higher gestational age than those with a prenatal diagnosis (mean, 0.4 weeks; $P=.01$; Figure 1 ). Thirty-seven subjects did not have specific gestational age reported but were classified as full term. These subjects were assigned the median gestational age among all subjects who were not preterm and whose gestational age was reported (38 weeks). Subjects without prenatal diagnosis exhibited a greater prevalence of nonchromosomal extracardiac congenital abnormalities in comparison with subjects with prenatal diagnosis $(9 \%$ vs $4 \%, P=.01)$. Fetal 
TABLE 1. Demographic factors and other descriptors by prenatal status

\begin{tabular}{|c|c|c|c|}
\hline & \multicolumn{2}{|c|}{$\begin{array}{c}\text { Prenatal diagnosis, } \\
\text { no. }(\%) * \text { or mean } \pm \text { SD }\end{array}$} & \multirow[b]{2}{*}{$P$ value* } \\
\hline & Yes $(n=677)$ & No $(n=228)$ & \\
\hline Female sex & $265(39)$ & $85(37)$ & .64 \\
\hline \multicolumn{4}{|l|}{ Race } \\
\hline White & $528(78)$ & $169(74)$ & .59 \\
\hline Black/African American & $99(15)$ & $37(16)$ & \\
\hline Asian & $16(3)$ & $6(3)$ & \\
\hline Other & $34(5)$ & $16(7)$ & \\
\hline Ethnicity & & & .28 \\
\hline Hispanic & 117 (17) & $49(21)$ & \\
\hline Non-Hispanic & $537(80)$ & $174(76)$ & \\
\hline Unknown & $23(3)$ & $5(2)$ & \\
\hline $\begin{array}{c}\text { Major extracardiac congenital } \\
\text { abnormality } \dagger(\mathrm{n}=904)\end{array}$ & $38(6)$ & $22(10)$ & .03 \\
\hline Chromosomal* & $10(1)$ & $2(1)$ & $.74 \S$ \\
\hline All other* & $28(4)$ & $20(9)$ & .01 \\
\hline Gestational age $(\mathrm{wk})$ & $37.8 \pm 1.8$ & $38.2 \pm 2.0$ & .01 \\
\hline Preterm & $100(15)$ & $31(14)$ & .66 \\
\hline Low birth weight $(<2500 \mathrm{~g})$ & $115(17)$ & $31(14)$ & .23 \\
\hline Birth weight $(\mathrm{g})$ & $3038 \pm 578$ & $3081 \pm 579$ & .34 \\
\hline
\end{tabular}

intervention on the atrial septum or aortic valve was reported in $26(3 \%)$ of the screened subjects. There were no differences in demographic factors, birth characteristics, presence of major extracardiac abnormalities, or incidence of preoperative death between those with fetal intervention and the remainder of the cohort.

\section{Center-Level Variation}

There was significant center-level variation in the proportion of subjects with prenatal diagnosis, ranging from $59 \%$ to $85 \%(P<.0001$, Figure 2$)$. The positive association between the size of the center's available screening population and the proportion of subjects with prenatal diagnosis is apparent. There was also significant center-level variation in mean fetal gestational age at diagnosis (range, 20.6-29.5 weeks; $P=.01)$.

\section{Risk Factors for Preoperative Mortality}

There were $26(3 \%)$ preoperative deaths. Univariable and multivariable associations between risk factors and preoperative death are presented in Table 2. Subjects who died before the Norwood procedure had significantly lower gestational age and birth weight and a much higher rate of extracardiac congenital anomalies compared with subjects who survived to the Norwood procedure. In addition, obstructed pulmonary venous return was more common among nonsurvivors $(23 \%$ vs $5 \%, P=.002)$.

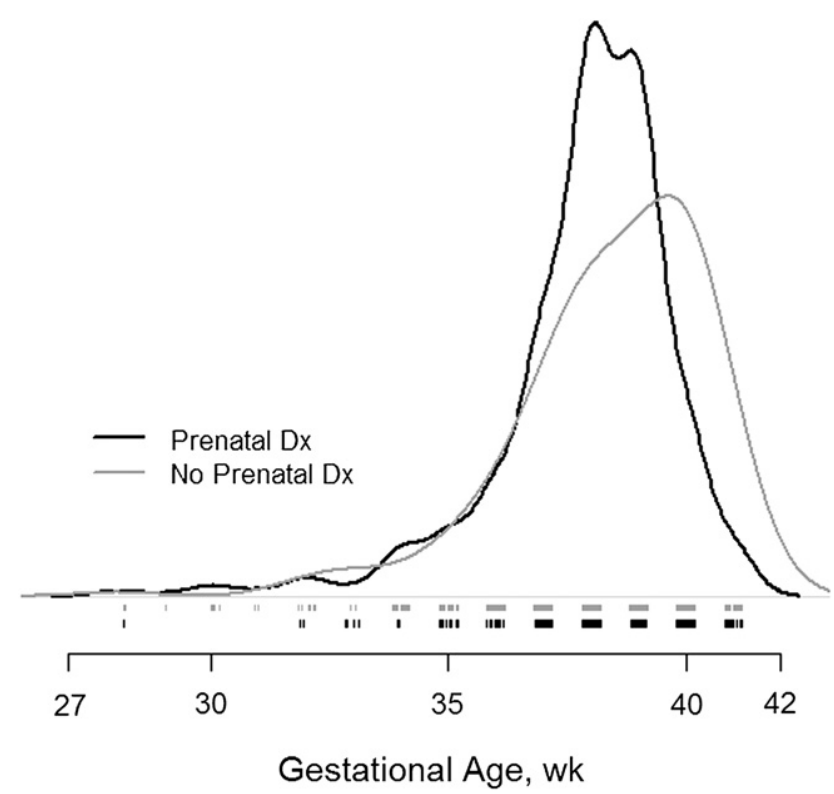

FIGURE 1. Kernel density estimates of gestational age according to prenatal diagnosis $(D x)$ status.

In multivariate logistic regression modeling, only major extracardiac congenital abnormalities, gestational age, and the presence or absence of obstructed pulmonary venous return remained significantly associated with preoperative mortality (Table 2). The presence of a noncardiac major congenital abnormality was most strongly associated with preoperative mortality $(P<.0001)$. The estimated odds of

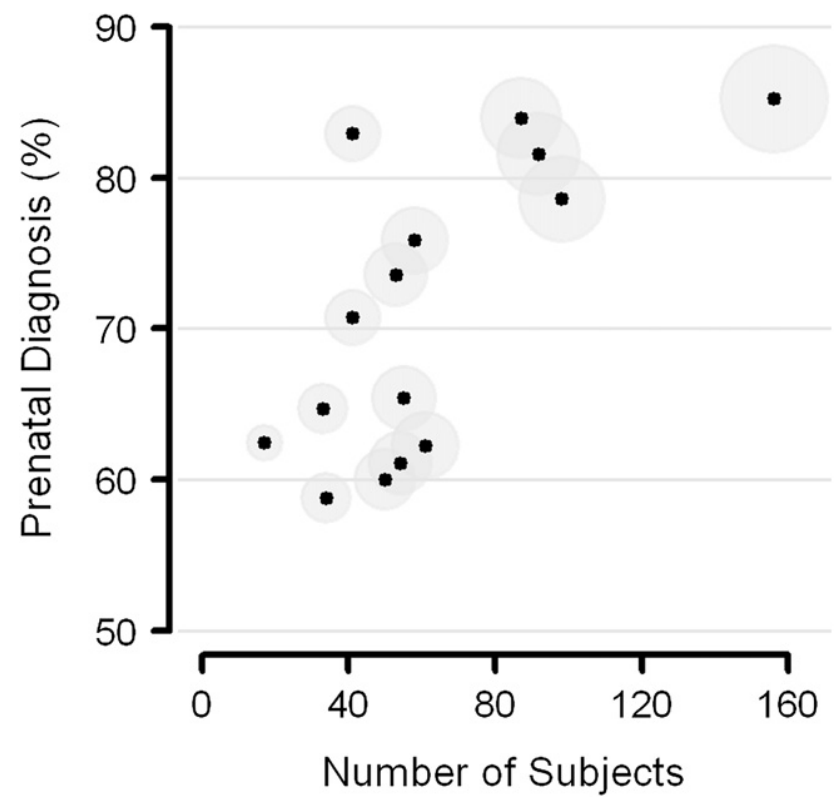

FIGURE 2. Center-specific proportions of subjects with prenatal diagnosis versus number of subjects screened $(\mathrm{n}=906)$. The 15 trial centers are displayed as circular symbols sized in relative proportion to the square root of their respective sample sizes. 
TABLE 2. Associations between risk factors and preoperative death

\begin{tabular}{|c|c|c|c|c|c|}
\hline & \multicolumn{3}{|c|}{ Unadjusted associations } & \multicolumn{2}{|c|}{ Multivariate model* } \\
\hline & Yes $(n=26)$ & No $(\mathbf{n}=\mathbf{8 8 0})$ & $P$ value $\dagger$ & Odds ratio $(95 \% \mathrm{CI})$ & $P$ value \\
\hline Prenatal diagnosis & $18(69 \%)$ & $659(75 \%)$ & 0.51 & & \\
\hline $\begin{array}{l}\text { Major extracardiac congenital } \\
\text { abnormality }\end{array}$ & $12(46 \%)$ & $48(6 \%)$ & $<.0001$ & $10.0(4.0-24.7)$ & $<.0001$ \\
\hline Chromosomal & $1(4 \%)$ & $11(1 \%)$ & .29 & & \\
\hline All other & $11(44 \%)$ & $37(4 \%)$ & $<.0001$ & & \\
\hline Gestational age (wk), mean \pm SD & $36 \pm 3$ & $38 \pm 2$ & .003 & $1.3(1.1-1.5)$ & .02 \\
\hline Low birth weight $(<2500 \mathrm{~g})$ & $11(42 \%)$ & $136(16 \%)$ & $<.001$ & & \\
\hline Aortic atresia & $16(62 \%)$ & $530(60 \%)$ & .90 & & \\
\hline Obstructed pulmonary venous return & $6(23 \%)$ & $44(5 \%)$ & .002 & $4.0(1.3-12.3)$ & .02 \\
\hline
\end{tabular}

$C I$, Confidence interval; $S D$, standard deviation. *Multiple logistic regression $(\mathrm{n}=905)$. Only risk factors displaying significant univariate association with mortality are included. $\dagger \chi^{2}$ Test of equal proportions.

preoperative mortality were 10 times greater for subjects with an extracardiac major congenital abnormality $(95 \%$ confidence interval, 4.1-24.8) after controlling for gestational age and obstructed pulmonary venous return. Prenatal diagnosis was not a significant correlate of outcome when added to this model.

\section{DISCUSSION}

The Single Ventricle Reconstruction Trial included systematic prospective screening data on the largest contemporary cohort of neonates with HLHS and related single right ventricular lesions with systemic outflow obstruction to date. This has permitted an unprecedented assessment of the timing and occurrence of prenatal diagnosis and risk factors for preoperative death.

\section{Characteristics Associated With Prenatal Diagnosis}

In contrast to data reported less than a decade ago with rates of prenatal diagnosis of less than $50 \%,{ }^{6,11}$ the majority $(75 \%)$ of neonates screened for this trial had a prenatal diagnosis. These infants were born at a significantly lower gestational age than subjects given diagnoses postnatally. However, although no difference was shown between diagnostic groups in the percentage born preterm (37 weeks' gestation), a considerably higher proportion of subjects in the postnatal diagnosis group were born at 39 weeks' gestation or later. The manner of delivery of subjects (natural labor, planned induction, or scheduled cesarean section) was not collected; however, we speculate that the overall difference in gestational age likely reflects a higher rate of planned induction of labor before 40 weeks' gestation among the prenatal diagnosis group.

There is increasing appreciation of the association of extracardiac and chromosomal abnormalities and congenital heart disease. ${ }^{12,13}$ In our study major extracardiac congenital abnormalities occurred less frequently in the prenatal diagnosis group than in the postnatal diagnosis group ( $6 \%$ vs $10 \%, P=.03)$, but interestingly, this difference was driven by nonchromosomal anomalies because the proportion of chromosomal anomalies was similar in both groups. Zyblewski and colleagues ${ }^{14}$ reported that women were 14 times more likely to terminate a pregnancy or seek comfort care for a fetus with congenital heart disease if a chromosomal abnormality was present. By extension, the presence of extracardiac structural anomalies diagnosed during prenatal ultrasound might also influence parental treatment decisions. The lack of association between chromosomal anomalies and prenatal diagnosis in our study might be explained by a low rate of prenatal karyotyping in this cohort; however, this is speculation because this information was not collected. We have observed in clinical practice that despite strong physician recommendations in favor of prenatal karyotyping, many women decline amniocentesis out of concern of potential risk to the pregnancy. ${ }^{14}$ It is important to note that differences in pregnancy termination rates in those with and without prenatal identification of more severe pathology might confound the association between prenatal diagnosis and preoperative mortality.

\section{Center-Level Variation}

Rates of prenatal diagnosis and timing (gestational age) of those diagnoses varied significantly by center. Figure 2 suggests a positive relationship between the number of subjects per site and the proportion of prenatal diagnoses. It seems plausible that patients receiving prenatal diagnoses whose parents have decided in favor of neonatal surgical palliation might be referred preferentially to large tertiary care centers with established fetal cardiology practices and well-known pediatric cardiac surgical programs. Center-level variation in gestational age at diagnosis could also be influenced by center variations in the number of patients choosing pregnancy termination or postnatal compassionate care only.

\section{Risk Factors for Preoperative Mortality}

Reports concerning the incidence and characteristics of preoperative mortality among infants with HLHS and 
related lesions are lacking. Mahle and associates ${ }^{6}$ found $4(1.8 \%)$ of 216 such subjects had preoperative death in a single-center series. In this multicenter cohort we found that $3 \%$ died before surgical intervention. Infants with a major extracardiac congenital anomaly had a 10-fold higher risk of preoperative death, largely because of the presence of nonchromosomal anomalies. This finding is consistent with previous reports. ${ }^{15}$ Previous reports suggest a positive relationship between prenatal diagnosis and improved preoperative management in patients with HLHS. ${ }^{7,16,17}$ However, we found no association between prenatal diagnosis and preoperative mortality. It is possible that improved education in neonatal units about the risks of ductaldependent congenital heart disease in neonates has resulted in earlier recognition of those not prenatally diagnosed. However, the inverse relationship between prenatal diagnosis and extracardiac congenital abnormalities found in the current study might confound the analysis of any relationship between prenatal diagnosis and preoperative mortality.

Low birth weight is frequently mentioned in clinical discussions as a risk factor for surgical outcome, a view borne out in an analysis of data on several types of congenital heart operations from the Society of Thoracic Surgeons Congenital Heart Database. ${ }^{18}$ Therefore we were surprised by the finding that gestational age, but not birth weight, was a significant predictor of preoperative mortality in multivariable analyses. We know from a recent analysis of growth parameters in the population screened for the Pediatric Heart Network's randomized controlled trial of enalapril versus placebo in infants with single-ventricle physiology that there is a significantly higher rate of growth retardation in infants with single-ventricle physiology compared with that seen in the general population. ${ }^{19}$ This suggests that, at a given gestational age, infants with single-ventricle physiology are smaller than infants with normal hearts. The physiological maturation implicit in increased gestational age might be more significant for fetal and neonatal well-being than size per se. The association between lower gestational age and increased mortality after infant heart surgery supports the importance of gestational age. ${ }^{20}$ This finding deserves further evaluation and consideration in risk stratification scores.

Obstructed pulmonary venous return is a well-known risk factor for postoperative mortality after the Norwood procedure. ${ }^{9}$ We found this also to be a risk factor for preoperative mortality. Moreover, our findings are consistent with previous studies that showed no benefit of prenatal diagnosis of atrial level restriction on outcome. ${ }^{21}$ Invasive decompression of the left atrium in fetuses with HLHS and a restrictive atrial septum has been reported ${ }^{22}$; whether this strategy will improve outcomes remains to be seen.

The limitations of this study are primarily related to potential selection bias. Only live subjects born at or referred to one of the 15 participating surgical centers were included.
These centers might not be representative of all centers performing complex neonatal surgery in North America. In addition, within the 15 trial centers, selection bias might have occurred as a result of variation in pregnancy termination after prenatal diagnosis. More detailed information on the causes of preoperative death was not available, and therefore some deaths might have been related to decisions not to pursue intervention. Finally, this study is restricted to events occurring before the Norwood procedure; assessment of the effect of prenatal diagnosis on postoperative survival and other outcomes awaits trial completion.

\section{SUMMARY}

Using prospective screening data on the largest contemporary cohort of neonates with HLHS and related single right ventricular lesions to date, we found that prenatal diagnosis by means of fetal echocardiographic analysis occurred in $75 \%$. Nonchromosomal major congenital anomalies were less common among those with a prenatal diagnosis. Preoperative death occurred in $3 \%$ of subjects and was independently associated with preterm birth, obstructed pulmonary venous return, and major extracardiac congenital abnormalities. Adjusted for gestational age and the presence or absence of obstructed pulmonary venous return, the estimated odds of preoperative mortality were 10 times greater for subjects with a major extracardiac congenital abnormality.

\section{References}

1. Allan LD, Crawford DC, Anderson RH, Tynan MJ. Echocardiographic and anatomical correlations in fetal congenital heart disease. Br Heart J. 1984;52: 542-8.

2. Chang RK, Chen AY, Klitzner TS. Clinical management of infants with hypoplastic left heart syndrome in the United States, 1988-1997. Pediatrics. 2002; 110:292-8.

3. Rasiah SV, Ewer AK, Miller P, Wright JG, Barron DJ, Brawn WJ, et al. Antenatal perspective of hypoplastic left heart syndrome: 5 years on. Arch Dis Child Fetal Neonatal Ed. 2008;93:F192-7.

4. Allan LD, Sharland GK, Milburn A, Lockhart SM, Groves AM, Anderson RH, et al. Prospective diagnosis of 1,006 consecutive cases of congenital heart disease in the fetus. J Am Coll Cardiol. 1994;23:1452-8.

5. Kumar RK, Newburger JW, Gauvreau K, Kamenir SA, Hornberger LK. Comparison of outcome when hypoplastic left heart syndrome and transposition of the great arteries are diagnosed prenatally versus when diagnosis of these two conditions is made only postnatally. Am J Cardiol. 1999;83:1649-53.

6. Mahle WT, Clancy RR, McGaurn SP, Goin JE, Clark BJ. Impact of prenatal diagnosis on survival and early neurologic morbidity in neonates with the hypoplastic left heart syndrome. Pediatrics. 2001;107:1277-82.

7. Tworetzky W, McElhinney DB, Reddy VM, Brook MM, Hanley FL, Silverman NH. Improved surgical outcome after fetal diagnosis of hypoplastic left heart syndrome. Circulation. 2001;103:1269-73.

8. Sano S, Huang SC, Kasahara S, Yoshizumi K, Kotani Y, Ishino K. Risk factors for mortality after the Norwood procedure using right ventricle to pulmonary artery shunt. Ann Thorac Surg. 2009;87:178-85.

9. Stasik CN, Gelehrter S, Goldberg CS, Bove EL, Devaney EJ, Ohye RG. Current outcomes and risk factors for the Norwood procedure. J Thorac Cardiovasc Surg. 2006;131:412-7.

10. Ohye RG, Gaynor JW, Ghanayem NS, Goldberg CS, Laussen PC, Frommelt PC, et al. Design and rationale of a randomized trial comparing the Blalock-Taussig and right ventricle-pulmonary artery shunts in the Norwood procedure. $J$ Thorac Cardiovasc Surg. 2008;136:968-75.

11. Fountain-Dommer RR, Bradley SM, Atz AM, Stroud MR, Forbus GA, Shirali GS. Outcome following, and impact of, prenatal identification of the candidates for the Norwood procedure. Cardiol Young. 2004;14:32-8. 
12. Gonzalez JH, Shirali GS, Atz AM, Taylor SN, Forbus GA, Zyblewski SC, et al. Universal screening for extracardiac abnormalities in neonates with congenital heart disease. Pediatr Cardiol. 2009;30:269-73.

13. Licht DJ, Shera DM, Clancy RR, Wernovsky G, Montenegro LM, Nicolson SC, et al. Brain maturation is delayed in infants with complex congenital heart defects. J Thorac Cardiovasc Surg. 2009;137:529-36.

14. Zyblewski SC, Hill EG, Shirali G, Atz A, Forbus G, Gonzalez J, et al. Chromosomal anomalies influence parental treatment decisions in relation to prenatally diagnosed congenital heart disease. Pediatr Cardiol. 2009;30:1105-11.

15. Wald RM, Tham EB, McCrindle BW, Goff DA, McAuliffe FM, Golding F, et al. Outcome after prenatal diagnosis of tricuspid atresia: a multicenter experience. $\mathrm{Am}$ Heart J. 2007;153:772-8.

16. Tibballs J, Cantwell-Bartl A. Outcomes of management decisions by parents for their infants with hypoplastic left heart syndrome born with and without a prenatal diagnosis. J Paediatr Child Health. 2008;44:321-4.

17. Verheijen PM, Lisowski LA, Stoutenbeek P, Hitchcock JF, Brenner JI, Copel JA, et al. Prenatal diagnosis of congenital heart disease affects preoperative acidosis in the newborn patient. J Thorac Cardiovasc Surg. 2001;121:798-803.
18. Curzon CL, Milford-Beland S, Li JS, O'Brien SM, Jacobs JP, Jacobs ML, et al. Cardiac surgery in infants with low birth weight is associated with increased mortality: analysis of the Society of Thoracic Surgeons Congenital Heart Database. J Thorac Cardiovasc Surg. 2008;135:546-51.

19. Williams R, Ravishankar C, Zak V, Atz A, Border W, Levine J, et al. Screening data from the Pediatric Heart Network Infant Single Ventricle Trial: association of single ventricle physiology with preterm birth and low birth weight. Congenit Heart Dis. 2008;5:96-103.

20. Costello JM, Polito A, Brown DW, McElrath TF, Graham DA, Thiagarajan RR, et al. Timing of delivery and outcomes in neonates with critical congenital heart disease. Circulation. 2009;120(suppl):S585.

21. Glatz JA, Tabbutt S, Gaynor JW, Rome JJ, Montenegro L, Spray TL, et al. Hypoplastic left heart syndrome with atrial level restriction in the era of prenatal diagnosis. Ann Thorac Surg. 2007;84:1633-8.

22. Vida VL, Bacha EA, Larrazabal A, Gauvreau K, Thiagaragan R, Fynn-Thompson F, et al. Hypoplastic left heart syndrome with intact or highly restrictive atrial septum: surgical experience from a single center. Ann Thorac Surg. 2007;84: 581-5.

\section{APPENDIX 1. Single Ventricle Reconstruction Trial participants}

National Heart, Lung, and Blood Institute: Gail Pearson, Victoria Pemberton, Rae-Ellen Kavey, Mario Stylianou, Marsha Mathis

Network Chair: University of Texas Southwestern Medical Center, Lynn Mahony

Data Coordinating Center: New England Research Institutes, Lynn Sleeper (PI), Sharon Tennstedt (PI), Steven Colan, Lisa Virzi, Patty Connell, Victoria Muratov, Lisa Wruck, Minmin Lu, Dianne Gallagher, Anne Devine, Thomas Travison, David F. Teitel

Core Clinical Site Investigators: Children's Hospital Boston, Jane W. Newburger (PI), Peter Laussen, Pedro del Nido, Roger Breitbart, Jami Levine, Ellen McGrath, Carolyn Dunbar-Masterson; Children's Hospital of New York, Wyman Lai (PI), Beth Printz (currently at Rady Children's Hospital), Daphne Hsu (currently at Montefiore Medical Center), William Hellenbrand, Ismee Williams, Ashwin Prakash (currently at Children's Hospital Boston), Ralph Mosca (currently at New York University Medical Center), Darlene Servedio, Rozelle Corda, Rosalind Korsin, Mary Nash; Children's Hospital of Philadelphia, Victoria L. Vetter (PI), Sarah Tabbutt (currently at the University of San Francisco), J. William Gaynor (Study Co-Chair), Chitra Ravishankar, Thomas Spray, Meryl Cohen, Marisa Nolan, Stephanie Piacentino, Sandra DiLullo, Nicole Mirarchi; Cincinnati Children's Medical Center, D. Woodrow Benson (PI), Catherine Dent Krawczeski, Lois Bogenschutz, Teresa Barnard, Michelle Hamstra, Rachel Griffiths, Kathie Hogan, Steven Schwartz (currently at the Hospital for Sick Children, Toronto), David Nelson; North Carolina Consortium: Duke University, East Carolina University, Wake Forest University, Page A. W. Anderson (PI; deceased), Jennifer Li (PI), Wesley Covitz, Kari Crawford, Michael Hines, James Jaggers, Theodore Koutlas, Charlie Sang, Jr, Lori Jo Sutton, Mingfen Xu; Medical University of South Carolina, J. Philip Saul (PI), Andrew Atz, Girish Shirali, Scott Bradley, Eric
Graham, Teresa Atz, Patricia Infinger; Primary Children's Medical Center and the University of Utah, Salt Lake City, Utah, L. LuAnn Minich (PI), John Hawkins, Michael Puchalski, Richard Williams, Linda Lambert, Jun Porter, Marian Shearrow; Hospital for Sick Children, Toronto, Brian McCrindle (PI), Joel Kirsh, Chris Caldarone, Elizabeth Radojewski, Svetlana Khaikin, Susan McIntyre, Nancy Slater; University of Michigan, Caren S. Goldberg (PI), Richard G. Ohye (Study Chair), Cheryl Nowak; Children's Hospital of Wisconsin, Nancy Ghanayem (PI), James Tweddell, Kathy Mussatto, Michele Frommelt, Lisa Young-Borkowski

Auxiliary Sites: Children's Hospital Los Angeles, Alan Lewis (PI), Vaughn Starnes, Nancy Pike; the Congenital Heart Institute of Florida (CHIF), Jeffrey P. Jacobs, MD (PI), James A. Quintessenza, Paul J. Chai, David S. Cooper, J. Blaine John, James C. Huhta, Tina Merola, Tracey Cox; Emory University, Kirk Kanter, William Mahle, Joel Bond, Leslie French, Jeryl Huckaby; Nemours Cardiac Center, Christian Pizarro, Carol Prospero; Julie Simons, Gina Baffa; University of Texas Southwestern Medical Center, Ilana Zeltzer (PI), Tia Tortoriello, Deborah McElroy, Deborah Town

Angiography core laboratory: Duke University, John Rhodes, J. Curt Fudge

Echocardiography core laboratories: Children's Hospital of Wisconsin, Peter Frommelt; Children's Hospital Boston, Gerald Marx

Genetics Core Laboratory: Children's Hospital of Philadelphia, Catherine Stolle

Protocol Review Committee: Michael Artman (Chair); Erle Austin; Timothy Feltes, Julie Johnson, Thomas Klitzner, Jeffrey Krischer, G. Paul Matherne

Data and Safety Monitoring Board: John Kugler (Chair); Rae-Ellen Kavey, Executive Secretary; David J. Driscoll, Mark Galantowicz, Sally A. Hunsberger, Thomas J. Knight, Holly Taylor, Catherine L. Webb 